\title{
ISSUES TO RESOLVE WITH THE USE OF EXTRACORPOREAL MEMBRANE OXYGENATION DURING INTERFACILITY TRANSPORTATION
}

\author{
Abstract \\ Extracorporeal membrane oxygenation (ECMO) support is indicated for patients \\ who are refractory to treatment, those with cardiogenic shock or respiratory \\ failure and those with exacerbations eligible for heart and lung transplantation. \\ The practitioner's experience and quantity of necessary resources are reasons why \\ regionalization could benefit these types of patients, establishing ECMO reference \\ centers and integrating a transportation network specialized in ECMO. \\ This type of transportation is a challenge for healthcare systems and physicians, \\ given its greater complexity, requiring a multidisciplinary and interterritorial \\ approach. \\ ECMO transportation is safer than without mechanical support, although there are \\ currently no criteria for starting the therapy on patients being transferred. Criteria \\ of lesser severity might be necessary for these patients. The training and \\ specialization of the team in extracorporeal support therapies, interfacility \\ transport and the systemization of the transfer can improve results. \\ There are no studies on the conditions that must be met by the transportation \\ media, although space and stability are important characteristics. Air transfer with \\ ECMO is an increasingly frequent option. Although there are data on its safety, \\ there are none on the physiology of patients undergoing ECMO at high altitudes, \\ data that could help with the indication and management of this type of \\ transportation.
}

\section{INTRODUCCIÓN}

La regionalización o centralización de determinados procesos, ha demostrado disminuir la morbimortalidad en determinadas patologías, como son el politraumatismo o los cuidados críticos neonatales (1-3). Actualmente está en discusión el beneficio de estos programas para otras patologías, como son los pacientes cardio-respiratorios (4), los pacientes subsidiarios de trasplante o de terapias de soporte vital extracorpóreo (Extracorporeal Life Support, ECLS) (7-12). La regionalización hace imprescindible el establecimiento de centros de referencia y de un transporte sanitario seguro $(2,5,6)$. Podría decirse que siempre y cuando se realice con seguridad, el traslado de pacientes críticos permite disminuir la morbimortalidad, al facilitar la llegada al centro útil (3). La composición del equipo viene dada por las necesidades del paciente (5), por tanto, el aumento de la complejidad de los pacientes a trasladar hace necesaria la formación y la especialización de los equipos de traslado. Por el momento, no existen datos suficientes que determinen un aumento de la supervivencia con el uso de equipos especializados de transporte (3), salvo en transporte pediátrico (13).

La mejora tecnológica y el aumento de la experiencia en técnicas de soporte vital avanzado y soporte vital extracorpóreo (ECLS), aumenta las posibilidades de tratamiento en el shock cardiogénico y distrés respiratorio agudo (SDRA) grave (14-22). La baja incidencia, la alta complejidad de manejo y la gran cantidad de recursos necesarios, hace necesaria la regionalización (7-11). 
A finales de los años noventa se comienzan a realizar traslados de pacientes pediátricos con insuficiencia respiratoria, observándose tasas de hasta un 39,1\% de mortalidad asociadas al traslado (23). En 2006 el estudio CESAR relanzó el interés por el empleo de la membrana de oxigenación extracorpórea (ECMO) en pacientes con SDRA al presentar unos resultados alentadores en cuanto a supervivencia de estos pacientes, pero una vez más, presentó relativa mortalidad relacionada con el traslado sin soporte extracorpóreo (14). Estudios realizados durante la pandemia de gripe $A\left(\mathrm{H}_{1} \mathrm{~N}_{1}\right)$ pudieron demostrar que el traslado de pacientes con SDRA grave es más seguro con ECMO que sin él, además de obtener similares resultados que los pacientes tratados desde el principio en el hospital de referencia $(10,24,25)$.

De similar manera evoluciona el transporte de pacientes con shock cardiogénico refractario. En 2013 nace en Paris el "Cardiac-RESCUE program" y tras él, son numerosos los consensos de expertos que recomiendan la estabilización hemodinámica con membrana de oxigenación extracorpórea venoarterial (ECMO VA) y posterior traslado al centro de referencia, sirviendo la ECMO VA como puente hacia la recuperación de la función cardiaca o hacia la asistencia ventricular y/o trasplante cardiaco $(11,18-22)$.

La expansión de la terapia y la universalización de los sistemas de transporte en ECMO es una realidad a falta estudios que establezcan los criterios estandarizados de soporte mecánico y las condiciones para realizar el transporte en ECMO. La creación de una red de transporte terrestre y aérea en ECMO conlleva el estudio regional de recursos hospitalarios así como de las infraestructuras hospitalarias disponibles, además de la creación de equipos de profesionales altamente cualificados.

\section{PROGRAMAS ECMO}

La baja incidencia de casos, la necesidad de formación especializada, la cantidad de recursos materiales y humanos necesarios, así como un posible efecto negativo en la morbimortalidad como consecuencia de un insuficiente volumen y experiencia, son razones para la centralización y por las que se plantean los programas de ECLS tanto para pacientes respiratorios como cardiológicos $(7-9,11)$.

Los hospitales de referencia favorecen la formación continuada y la experiencia recibida por un volumen aceptable de pacientes al año (9). La integración de diferentes servicios es la pieza angular del abordaje multidisciplinar. Mientras que la relación con los hospitales no referentes y proveedores de pacientes asienta el programa, tanto por la labor educacional a la hora de la detección precoz de este tipo de pacientes, como por el refuerzo positivo generado por la autoevaluación $(7,9)$. Los equipos ECMO, son equipos especializados móviles que valoran la necesidad de un transporte especializado con o sin ECMO a los centros de referencia, permitiendo la universalización del programa $(7,10,25-27)$.

Se llama transporte en ECMO Primario cuando es el equipo del hospital de referencia quien se traslada, valora e implanta el soporte para el traslado y el transporte Secundario, cuando la terapia se inicia en el hospital de origen y es trasladado a un centro de referencia ya sea por uno u otro equipo (27). Este tipo de sistema precisa de mayor disponibilidad de recursos, formación y experiencia en ECMO, siendo un programa establecido y más avanzado.

El aumento de pacientes trasladados en ECMO a centros de referencia se ha llegado a multiplicar por cinco desde el 2011 en algunas regiones, potenciado por la 
existencia de estos programas (28). Son necesarios futuros estudios sobre el costeeficiencia de estos programas comparados con aquellos sin coordinación establecida (12).

\section{EQUIPO DE TRANSPORTE EN ECMO}

Los equipos de transporte deben estar coordinados por el centro de referencia y sus componentes deberían de tener la formación y la experiencia continuada en el manejo del paciente en ECMO $(7,10,26,27)$, así como estar entrenado y habituado al transporte de pacientes críticos, y en caso de realizar un transporte aéreo, estar específicamente formado en ello (28). La formación en transporte en ECMO puede ser determinante ya que puede a ayudar a controlar los efectos adversos más graves, dependientes de fallos del material en un ambiente hostil (29).

Existen diferentes configuraciones del equipo en función de la experiencia del centro y en función del tipo de traslado a realizar.

Las diferentes responsabilidades del proceso deben quedar bien establecidas, existiendo $(27,30)$ :

1. Médico Intensivista:

- Coordinador del traslado, se encarga de coordinar con ambos centros el proceso.

- Toma la responsabilidad del paciente. Evalúa clínicamente al paciente e indica, o no, la terapia con ECMO. Informa a la familia y firma el consentimiento informado.

- Toma las decisiones en torno al paciente en ECMO hasta la llegada al hospital de referencia (programación de los flujos de sangre y aire de la ECMO, dosis de anticoagulación, fluidoterapia, vasopresores, etc).

2. Médico responsable de la canulación: en algunos centros es el cirujano cardiovascular, en otros es el intensivista formado en canulación percutánea.

3. Especialista en ECMO: perfusionista, médico o enfermero/a:

- Material del circuito ECMO.

- Material necesario en el hospital de origen para el procedimiento.

- Cebado de la máquina.

- Manejo del circuito ECMO durante el traslado.

4. Enfermero/a especialista en Cuidados Intensivos.

- Cuidado de enfermería del paciente.

○ Administración de medicaciones, fluidoterapia, sangre, etc.

5. Especialista en transporte y respiratorio:

- La ventilación mecánica, conexiones y disposición de gases.

- Esta función debe ser capaz de asumirla el médico responsable del traslado en caso de no haber espacio suficiente para el personal en el medio de transporte.

Por poner algunos ejemplos: El hospital Karolinska (Estocolmo), tiene un equipo formado por un cirujano, un intensivista con experiencia en ECMO, un perfusionista y en ocasiones un enfermero/a especialista añadido (30). La Universidad de Columbia (Nueva York) tiene un equipo formado por un cirujano cardiotorácico, un fellow de cirugía, dos perfusionistas y dos paramédicos especialistas en paciente crítico (31). En la Guayana francesa, el equipo está formado por un cirujano cardiovascular, un médico del SMUR con o sin ayuda de un médico intensivista y/o perfusionista (32). 


\section{CRITERIOS DE ECMO PARA TRASLADO EN LA INSUFICIENCIA RESPIRATORIA AGUDA}

Recientes revisiones sobre el manejo del SDRA grave sitúan a la ECMO como terapia de rescate en pacientes con hipoxemia refractaria a terapia ventilatoria optimizada y otras medidas como el prono. No existen por el momento recomendaciones unificadas por falta de evidencia significativa, a la espera del estudio en activo EOLIA (NCT01470703), con el cual se espera poder delimitar mejor los criterios de ECMO en el SDRA $(12,16,33)$. Las guías de la Extracorporeal Life Support Organization (ELSO) recomiendan considerar la ECMO con $\mathrm{PaO}_{2} / \mathrm{FiO}_{2}$ $<150$ y la indican con $\mathrm{PaO}_{2} / \mathrm{FiO}_{2}<100$ o acidosis respiratoria con $\mathrm{P}_{\text {plateu }}>30 \mathrm{~cm} \mathrm{HO}$ (34). Estos criterios contrastan con recientes revisiones que se mantienen expectantes y recomiendan la ECMO en los casos de SDRA más graves (16).

En lo que sí parece haber más acuerdo es en la precocidad del tratamiento, ya que sí disminuye la mortalidad al implantarla durante el primer o segundo día de refractariedad al tratamiento ventilatorio optimizado $(34,25)$.

El traslado de pacientes con insuficiencia respiratoria moderada-grave a centros de referencia en ventilación mecánica y ECMO, supone un alto riesgo, mayor en aquellos con hipoxemia grave y/o acidosis respiratoria. El traslado en ECMO ha demostrado ser un traslado más seguro para estos pacientes que sin soporte extracorpóreo $(10,24,25)$, hasta el punto de cuestionarse si deben existir o no unos criterios específicos de ECMO en pacientes a trasladar.

El procedimiento utilizado en el estudio ECMOnet instauraba la ECMO utilizando criterios de menor gravedad en aquellos pacientes a trasladar: $\mathrm{PaO}_{2} / \mathrm{FiO}_{2}<70$ y PEEP $>15$ si se encontraban en el centro de referencia y con $\mathrm{PaO}_{2} / \mathrm{FiO}_{2}<100$ con PEEP > 10 si eran pacientes a trasladar $(10,23)$. A medida que ha aumentado la experiencia, se han ido ajustando los criterios de inclusión. En un estudio posterior, una $\mathrm{PaO}_{2} / \mathrm{FiO}_{2}<85$ y/o acidosis respiratoria con $\mathrm{pH}<7,2$ eran criterios de activación del protocolo y valoración por el equipo ECMO. Tras la optimización de la ventilación mecánica y maniobras de rescate respiratorio, el 13\% de los pacientes pudieron trasladarse de forma segura sin ECMO cumpliendo los criterios: $\mathrm{pO}_{2}>90 \mathrm{mmHg}$ con $\mathrm{FIO}_{2} 1, \mathrm{PCO}_{2}<65$ mmHg y $\mathrm{PEEP}<20$ (25).

Un reciente metanálisis que incluye un total de 1481 pacientes transportados en ECMO, extraídos de estudios heterogéneos realizados entre 1994 y 2016, contrasta con lo anterior presentado, siendo el grado de hipoxemia media pre-ECMO de $\mathrm{PaO}_{2} / \mathrm{FiO}_{2}$ : 59 y presentando una supervivencia de alrededor del 60\% (35). Con todo ello no queda más que esperar a futuros estudios, siendo prudentes a la hora de trasladar un paciente con SDRA grave, valorando la precocidad del traslado y los posibles criterios de menor gravedad para la implantar la ECMO debido al alto riesgo que supone un transporte.

\section{CRITERIOS DE ECMO PARA TRASLADO EN EL SHOCK CARDIOGÉNICO.}

Las últimas recomendaciones sobre el manejo del shock cardiogénico, incluyen las técnicas de soporte circulatorio mecánico en caso de refractariedad al tratamiento habitual con un grado de recomendación IIb (20).

La escala INTERMACS (Interagency Registry for Mechanical Assisted Circulatory Support) estratifica la gravedad del fallo cardiaco y su mortalidad, permitiendo hacer una correcta elección de los pacientes que se beneficiarán de uno u otro dispositivo de asistencia circulatoria mecánica, así como establecer un pronóstico tras un trasplante cardiaco urgente (tabla 1$)(20,36,37)$. 
En caso de shock cardiogénico refractario en estadio 1 y 2 de la escala INTERMACS, se recomienda el uso de dispositivos de soporte circulatorio a corto plazo como puente a la recuperación miocárdica o a la valoración de implantación de dispositivos de larga duración y/o trasplante cardiaco (12-22,37).

La experiencia del equipo es clave, eligiendo el momento adecuado para su implantación, pudiendo caer en la futilidad por instaurarlo demasiado temprano como demasiado tarde (21). Escalas como el SAVE-score, pueden ser de ayuda a la hora de valorar la implantación de estos dispositivos (38).

Es cierto que está muy entredicho cual es el dispositivo ideal de soporte en fase aguda $(12,18,39)$, ya que la falta de descarga completa ventricular puede empeorar la situación o mantener una fase de fallo multiorgánico. Entre los dispositivos percutáneos se encuentran: el balón de contrapulsación intra-aórtico (BIAC), la ECMO VA periférica y otros nuevos como el Impella ${ }^{\circledR}$ o Tandem Heart ${ }^{\circledR}$. Estos dos últimos, se colocan mediante arteriografía, el Impella ${ }^{\circledR}$ únicamente ofrece soporte ventricular izquierdo y el Tandem Heart ${ }^{\circledR}$ biventricular $(12,18,39)$. La experiencia por el momento es baja, el coste con respecto a la ECMO es mayor, pueden no aportar el suficiente soporte hemodinámico en los pacientes más graves (12), y son difíciles de implementar en los programas de transporte primario. En cuanto al BIAC ya no se recomienda de forma rutinaria en el shock cardiogénico (18), aunque sí hay estudios que apoyan su uso concomitante a la ECMO VA al mejorar la hemodinámica y disminuir el riesgo de EAP $(40,41)$.

No existen estudios prospectivos exclusivamente de transporte de pacientes con shock cardiogénico. Son a destacar los estudios retrospectivos realizados en islas francesas donde se analizan transportes en ECMO VA de largas distancias (136km$10.000 \mathrm{~km})$ a los centros de trasplante cardiaco $(32,42,43)$. En el estudio más reciente, de los pacientes a evacuar el 57,6\% lo hizo con ECMO-VA y con balón de contrapulsación asociado en el 78,6\% de ellos. Los pacientes trasladados con soporte circulatorio, presentaron supervivencias similares a aquellos en los que la ECMO se instauraba en el hospital de referencia (36,8\% vs 37\%) y con resultados post-trasplante cardiaco similares a aquellos que fueron transportados sin soporte circulatorio (85,7\%-83,3\%), pese a la mayor gravedad de estos primeros (43).

En cuanto a las asistencias ventriculares (VAD) permiten un soporte hemodinámico completo a medio plazo como puente al trasplante cardiaco (20). Así mismo, también puede ser utilizado este soporte como puente a la recuperación, sobre todo en pacientes jóvenes con patologías potencialmente reversibles, como miocarditis o miocardiopatía postparto (20). La implantación de VAD en el centro hospitalario de origen para luego ser trasladado al centro de referencia en trasplante cardiaco está descrita, requiere alta experiencia de ambos centros y una evaluación previa del paciente como candidato a trasplante $(44,45)$.

\section{OTRAS POSIBLES APLICACIONES DEL TRASLADO EN ECMO}

Resucitación Cardiopulmonar con ECMO en medio extra hospitalario:

La resucitación cardiopulmonar con ECMO (ECPR) podría ofrecer mejores resultados neurológicos que la reanimación cardiopulmonar convencional (RCP) y contribuir a la donación de órganos de aquellos que fallecen $(12,46-48)$. Pero por el momento los resultados de estudios controlados y prospectivos son pobres $(12,49,50)$. La importancia de recibir RCP de calidad de forma inmediata, sumado a la elección precisa del paciente y a la precocidad de la terapia ECMO (48), han llevado a realizar estudios sobre el beneficio de su implantación en el ambiente 
extra hospitalario. Ante posibles resultados futuros favorecedores a la realización de estas técnicas en medio extra hospitalario, cabe preguntarnos qué podría suponer y si estamos preparados para ello.

Donación en Asistolia Controlada:

La implantación de la ECMO VA en donación en asistolia controlada (DAC) ha demostrado mejores resultados que la extracción de órganos ultrarrápida $(51,52)$. El desplazamiento de un equipo móvil de coordinadores de trasplante, junto con el material y el personal necesario al hospital de origen, ya está descrito (53), de tal manera que se podría ofrecer la DAC en hospitales donde no existe este recurso.

Pacientes en lista de trasplante pulmonar reagudizados:

El traslado de pacientes en lista de trasplante pulmonar con insuficiencia respiratoria reagudizada a centros de referencia en trasplante, se consideran traslados de alto riesgo y sería necesario que lo llevaran acabo personal especialista con los medios necesarios. La intubación y conexión a ventilación mecánica está asociada con pobres resultados en el post-trasplante de pulmón. La ECMO y los eliminadores extracorpóreos de $\mathrm{CO}_{2}\left(\mathrm{ECCO}_{2} \mathrm{R}\right)$ implantados en el paciente despierto como puente al trasplante de pulmón, ha demostrado mejorar la supervivencia de tipo de pacientes. $(16,34)$. En cuanto al traslado en ECMO de pacientes despiertos, de momento la evidencia se limita a la descripción de casos con buen resultado (55).

\section{TRASLADO EN ECMO: ELECCIÓN DEL MEDIO DE TRANSPORTE}

Esta cuestión es muy específica de cada uno de los programas ECMO y es conveniente que cada país estudie su propia situación. La elección de uno u otro medio depende de muchas variables: distancias a recorrer, orografía del terreno, nivel de carreteras, estructuras hospitalarias, meteorología dominante en la región y disponibilidad de medios de transporte adecuados con la tecnología adecuada $(33,56,57)$.

Las recomendaciones sobre el transporte a utilizar son muy variables, en las guías inglesas de transporte crítico, recomiendan considerar el traslado con helicóptero para distancias por encima de los $80 \mathrm{~km}$ y avión medicalizado por encima de los $240 \mathrm{~km}$ (57), mientras que las guías ELSO recomiendan el transporte terrestre hasta $300 \mathrm{~km}$, helicóptero hasta $600 \mathrm{~km}$ y avión por encima de estas cifras $(27,58)$. Es importante estudiar la evolución en aquellos países donde se realizan numerosos traslados de pacientes críticos y en ECMO, no evolucionando de forma similar por otros condicionantes como son la meteorología y la noche. Así el Hospital Karolinska, que pese a tener las estructuras hospitalarias necesarias, su transporte en ECMO se basa en un transporte terrestre para distancias menores $300 \mathrm{~km}$ y avión para distancias mayores, el cual es utilizado para el 60\% de los traslados (29). En zonas de Europa con otras condiciones, sí es cierto que ha aumentado el transporte interhospitalario crítico en helicóptero gracias a la adaptación de las infraestructuras y de la introducción de helicópteros con mejores prestaciones, estudios alemanes de transporte en ECMO comentan traslados en helicóptero entre 60 km-178km (59).

\section{TRANSPORTE TERRESTRE:}

El transporte en ambulancia es el medio más utilizado para el traslado sanitario, en un reciente metanálisis se estimó que un 53\% de los traslados en ECMO son en ambulancia (35). Las principales ventajas de este tipo de transporte son su disponibilidad, su coste y su utilización en prácticamente casi todas las situaciones (27). La disponibilidad de ambulancias para traslados de pacientes complejos que 
requieren mucha cantidad de material y personal, es relativa, siendo cuestionable que las ambulancias convencionales sean el tipo de transporte óptimo para este tipo de traslados. El Hospital Karolinska elige un autobús medicalizado para el transporte en ECMO. La necesidad de transportes terrestres voluminosos aumentan los tiempos de traslado de ida y vuelta, por ello algunos centros utilizan un vehículo rápido con el personal y el material. Otro aspecto a valorar es la importancia del estado de las carreteras a recorrer, ya que carreteras tortuosas aumentaran los tiempos de traslado y serán más incómodas a la hora de trabajar. TRANSPORTE EN HELICÓPTERO:

El estudio de Mendes et al cifra en un 22\% los traslados que se realizan en helicóptero (35). El helicóptero presenta como principales ventajas la velocidad, la estabilidad del medio de transporte y el traslado de cama a cama sin otros transportes intermediarios, todo ello hace pensar que es el medio ideal para el traslado de pacientes críticos.

En cuanto al tipo de helicóptero a utilizar, es importante analizar el equilibrio entre el espacio en la cabina y peso máximo al despegue acorde con las infraestructuras disponibles, siendo además importantes; la velocidad crucero, datos de estabilidad, ruidos y vibraciones. El tiempo de autonomía es importante aunque no se realicen largas distancias, ya que amplia el rango de posibilidades y disminuir tiempos.

Los helicópteros utilizados para el traslado en ECMO, deben ser espaciosos y que admitan un peso variable aproximado entre $500 \mathrm{~kg}$ (los equipos más reducidos) y $1000-1200 \mathrm{~kg}$ (2 pilotos, equipo ECMO completo, paciente y material). Helicópteros como el EC-145, Bell 429, Bell 412 o el reciente AW 169, presentan buenas prestaciones con pesos máximo al despegue más limitado. Helicópteros utilizados son el AW 139 o el Sykorsky S76, amplios y estables, pero más costosos, pesados y con necesidad de infraestructuras menos accesibles.

Como principales inconvenientes al traslado con helicóptero son la necesidad de actualizar las infraestructuras hospitalarias y la necesidad de implementar la tecnología aeronáutica que permita la nocturnidad y los vuelos con mala meteorología.

En cuanto al coste-eficacia, no existe por el momento ningún estudio específico del transporte en ECMO y los existentes, son difícilmente comparables por los diferentes modelos de financiación. La mayor coste-eficiencia del transporte aéreo medicalizado depende de la suma de varios criterios: distancias moderadas (80$350 \mathrm{~km}$ ), traslado directo sin necesidad de transporte terrestre, personal médico altamente cualificado, sumado a una correcta gestión y elección del tipo de paciente a trasladar $(6,56,57)$.

\section{TRANSPORTE EN AVIÓN}

El avión presenta como ventajas la estabilidad, la amplitud, la presurización constante, su autonomía, la ausencia de limitaciones de peso, ni de meteorología, ni de nocturnidad. Grandes desventajas son el depender de aeropuertos con la logística que ello conlleva y la necesidad de un traslado previo y posterior en ambulancia $(27,56,57)$. Un $27 \%$ de los traslados se han realizado con este medio, permitiendo traslados en ECMO de largas distancias, siendo un 7\% de los traslados totales internacionales (35).

\section{EQUIPAMIENTO Y PREPARACIÓN DEL TRASLADO}

La planificación del transporte en ECMO incluye una revisión de todo el material necesario, comprobando la compatibilidad con el medio de transporte elegido 
(conexiones eléctricas, voltajes, fijaciones). Es preciso conocer todo el material con su tiempo de autonomía y la posibilidad o no de cambio de baterías, siendo recomendable disponer de una fuente eléctrica y con capacidad para todo el material. En caso de transporte aéreo, los aparatos deben estar certificados por Aviación Civil para el modelo específico de avión o helicóptero, así como ajustar los aparatos electromagnéticos para evitar interferencias durante el vuelo $(1,26$ 28,56,57).

Cada programa ECMO diseña su propio equipo de material, en función del tipo de misiones a realizar y de su experiencia. Se recomienda la realización de mochilas y de una lista de chequeo que ayude a disminuir los riesgos de olvidos de material (Tablas 2 y 3: modelos de Checklist adaptables). $(27,29,30,60,61)$.

Se debería disponer de material para una monitorización avanzada (PAI, , pulsioximetría, BIS, capnografía, PCP y oximetría cerebral si fuera preciso) disponer de sangre, plaquetas y plasma, nevera, dos equipos quirúrgicos, material de canulación y doble membrana de ECMO. Podría ser de utilidad principalmente en traslados primarios en ECMO: compresor torácico, ecógrafo portátil, radiometer de transporte, control de coagulación y hemoglobina. Es necesaria una camilla de traslado apta para este tipo de pacientes y con capacidad para fijar todo el material $(26,27,56)$. Existen en el mercado camillas especialmente diseñadas para el traslado en ECMO, con la idea de dejar el material fijado y sin necesidad de colocar el aparataje dentro del medio de transporte.

El ventilador mecánico y el monitor de transporte deberían ofrecer las prestaciones más avanzadas sin olvidar su portabilidad, existen ya en el mercado ventiladores mecánicos de transporte adaptados a la presión barométrica. Esta prestación, junto con la portabilidad y atenuación de vibraciones, también es ofrecida para balones de contrapulsación de transporte. Existen varios tipos de dispositivos ECMO utilizados en transporte, es importante conocer el peso y el volumen que ocupan, así como las especificaciones técnicas, ya que no todos ofrecen las mismas prestaciones. Es recomendable que el peso de todo el material necesario para la canulación y la ECMO, no exceda los $35 \mathrm{~kg}$ en caso de realizar un transporte aéreo (32).

NECESIDAD DE AIRE Y OXÍGENO:

Es importante conocer la cantidad de oxígeno y aire comprimidos necesarios, contando siempre con posibles contratiempos que aumenten el tiempo de traslado. Los problemas de logística y el quedarse sin aporte de oxígeno son incidentes relativamente frecuentes (29).

Un paciente en ECMO o en asistencia ventricular (VAD) con una membrana de oxigenación incorporada, tendrá el gasto del respirador mecánico y el de la membrana, debiendo conocer ambos volúmenes/minuto. El dispositivo ECMO durante el transporte y durante la mayoría de la terapia VV, estará configurado con $\mathrm{FiO}_{2}$ 1, siendo el sweep air (de 0 a $10 \mathrm{lpm}$ ) el volumen/minuto de la ECMO, el cual se configurará en función de las necesidades ventilatorias (normalmente una relación 1:1 con el flujo de sangre) (34).

Los ventiladores mecánicos de transporte de tipo turbina, permiten realizar las mezclas de oxígeno con el aire ambiente, siendo independientes de una toma de aire comprimido, lo cual nos hace prescindir de este tipo de botellas. Hay que contar con un gasto extra de gases que el respirador utiliza para control interno y que suele ser entre $100-500 \mathrm{ml} / \mathrm{min}(62,63)$. Es aconsejable que la $\mathrm{FiO}_{2}$ del 
respirador sea la menor que asegure una correcta oxigenación y que la ventilación mecánica sea protectora o ultra protectora (64).

Una vez conozcamos las necesidades de aire y oxígeno, así como el tiempo previsible de traslado, podremos calcular el número de botellas necesarias (65)(material complementario tabla 6 y 7). Durante el traslado es importante vigilar el gasto y los litros disponibles, pudiendo adelantarnos ante cualquier problema de este tipo. Existen en el mercado, botellas de oxígeno medicinal con pantalla digital informando del tiempo disponible sin necesidad de realizar cálculos con el manómetro.

\section{PLANIFICACIÓN DEL TRASLADO}

Los problemas de logística suelen conllevar aumento del tiempo de traslado y riesgos, documentándose aumento de las infecciones nosocomiales y más efectos adversos durante los traslados de largas distancias $(29,43)$. Por ello, el trabajo de sistematizar el transporte puede ayudar a mejorar los resultados.

Es aconsejable contar con posibles retrasos y realizar un plan de actuación secundario, conociendo las características y la localización de hospitales alternativos. Es importante la compenetración del equipo de traslado y médico. En caso de transporte aéreo, la realización de un plan de vuelo junto a los pilotos, disponiendo de la información acerca de los helipuertos de referencia, áreas de toma urgente durante nuestro recorrido, aéreas de combustible y el establecimiento conjunto de la altura media y máxima de vuelo en función de la situación clínica del paciente.

\section{PECULIARIDADES DEL TRANSPORTE AEREO}

Durante el vuelo se deberán tomar ciertas medidas contra la hipotermia, el sonido, las vibraciones y las energías de aceleración y deceleración. La mejoría tecnológica sanitaria y aeronáutica, minimiza de forma importante estos problemas y por el momento, no existen descritas complicaciones secundarias a ello $(29,32)$. Otro factor que puede afectar a pacientes respiratorios y pediátricos es la escasa humedad (aproximada al 10\%) (66), pudiéndose tomar medidas de humificación activa en pacientes de muy alto riesgo.

La gran influencia del traslado aéreo que podríamos esperar en nuestro paciente es secundaria a la disminución de la PalveolarO $2\left(\mathrm{PAO}_{2}\right)$ y al aumento del volumen de los gases a medida que disminuye la presión con la altura (66-69). La documentación de complicaciones relativas a la hipoxemia secundaria a la disminución de la presión alveolar de oxígeno $\left(\mathrm{PAO}_{2}\right)$, es baja (3-4\%) (32). No existe hasta la fecha ningún estudio específico sobre la fisiología del paciente en ECMO ante los cambios de altura, siendo importante para la seguridad del traslado así como para individualizar el plan de vuelo. A alturas mayores de vuelo, se disminuye el consumo del helicóptero, lo cual aumentaría la autonomía y con ella, las posibilidades de transporte sin paradas extraordinarias y mejora del costeeficiencia.

En altura los volúmenes-minuto aumentan tanto en la ECMO, como en los respiradores, existiendo el riesgo de hiperventilar a nuestro paciente (66-69). Cada vez son más los aparatos adaptados al transporte aéreo, modificándose en función de la presión barométrica, como son algunos ventiladores mecánicos y balones de contrapulsación, pero por el momento las membranas de oxigenación extracorpóreo no ofrecen esta prestación. Es posible realizar cálculos basados en la Ley de Boyle-Mariotte para conocer qué efectos sobre la ventilación generará la 
altura (material complementario tabla 6 y 8). Este es uno de los motivos por los que puede ser recomendable un radiometer de transporte.

Así mismo, podemos calcular la $\mathrm{FiO}_{2}$ a la que deberemos aumentar el respirador a determinada altura, pudiendo individualizar el plan de vuelo o contraindicar un traslado aéreo (material complementario tabla 8 y 9).

\section{COMPLICACIONES DURANTE EL TRASLADO}

La mortalidad durante el traslado en ECMO es prácticamente nula en comparación con los traslados sin soporte extracorpóreo $(23-25,29,35)$. Merece la pena comentar un estudio recientemente publicado, donde específicamente se evalúan los efectos adversos producidos en 514 transportes con ECMO. Hay que decir, que la mayoría de estos traslados fueron primarios y un 59\% en avión, lo cual puede reflejar la complejidad de los casos analizados. En este estudio, la probabilidad de sufrir un efecto adverso fue de un 40\%, siendo mayor en aquellos traslados superiores a $3 \mathrm{~h}$. En las tablas 4 y 5 se muestra la clasificación realizada de los eventos adversos, así como las frecuencias con las que se registraron. (29)

A raíz de estos resultados, podemos decir que la mayoría de los eventos adversos que acontecen durante el transporte en ECMO son relativos al paciente (29), y que generalmente se tratan de incidentes habituales durante las primeras horas de inicio de una ECMO. Un equipo con experiencia en técnicas de soporte extracorpóreo suele estar concienciado y entrenado en estos temas, manteniendo un margen de respuesta mayor. En cambio, la mayor parte de los eventos adversos responsables de riesgos con consecuencias inminentes, son derivados de problemas con el equipamiento médico (29) y con la acción del transporte en sí, con los que el personal sanitario no está tan familiarizado. Así mismo, llama la atención que el 19\% de los riesgos inminentes sean por fallos del personal médico, principalmente debidos a problemas de comunicación (29). Todo ello nos lleva a pensar que es necesaria una formación específica en el traslado de pacientes críticos y en ECMO, abordando tanto cuestiones médicas y técnicas como de trabajo en equipo (70).

\section{CONCLUSIONES}

El soporte extracorpóreo con ECMO está indicado en casos de refractariedad al tratamiento en casos de shock cardiogénico e insuficiencia respiratoria, así como, en aquellos pacientes reagudizados subsidiarios de trasplante cardiaco y pulmonar. La experiencia y volumen necesarios para obtener buenos resultados, junto a la gran cantidad de recursos que se precisa, son causas por las que se creen necesarios la realización de programas de soporte extracorpóreo, coordinados por los que los centros de referencia en ECMO y centros de trasplante cardiopulmonar, integrando una red transporte especializado en ECMO.

Es escasa la evidencia sobre los criterios exactos en los que se debe alertar al equipo de traslado y los criterios de soporte mecánico en condiciones de traslado. Son cuestiones a valorar la seguridad del paciente y el coste-eficiencia del sistema, siendo necesarios futuros estudios sobre la utilidad de estos programas.

Un equipo especializado en ECMO es clave para la valoración clínica y para la seguridad del paciente durante el traslado. El conocimiento tecnológico en condiciones de transporte y la formación específica del equipo en transporte crítico, es importante para afrontar fallos del equipamiento que puedan suponer un alto riesgo inminente. 
La imposibilidad de aplicar los estudios de coste-efectividad sobre los medios de transporte por cuestiones inherentes a cada región y sistema, hacen que se requiera un análisis específico individual. El helicóptero, por su velocidad, la posibilidad de recorrer largas distancias sin necesidad de otros medios y por su estabilidad, podría ser el medio de transporte más adecuado en distancias medias, siempre y cuando estén disponibles las infraestructuras hospitalarias, los medios aeronáuticos necesarios y las condiciones climatológicas lo favorezcan. A pesar de que se ha documentado la seguridad de este tipo de traslado, aun es necesario estudiar la fisiología de la ECMO en altura para aumentar la seguridad durante el traslado y adaptar el plan de vuelo al paciente. El medio terrestre sigue siendo el transporte más utilizado, por su disponibilidad y su coste, pero conviene destacar la necesidad de adaptar la ambulancia al traslado de pacientes críticos y ECMO. En cuanto a los traslados de largas distancias y en avión, conllevan más incidencias logísticas y médicas, pero hacen posible la oferta de este recurso a las regiones más remotas con un análisis beneficio-riesgo positivo.

El traslado en ECMO supone un reto para el sistema sanitario, no solo por la complejidad técnica o médica que este conlleva, sino porque supone la creación de una estructura que puede sentar las bases de la relación multidisciplinar en el ámbito extra hospitalario y aumentar la relación interhospitalaria en pro del paciente.

\section{BIBLIOGRAFIA}

1. Blakeman TC, Branson RD. Inter- and Intra-hospital Transport of the Critically Ill. Respiratory Care. 2013;58:1008-23.

2. Iwashyna TJ, Christie JD, Kahn JM, Asch DA. Uncharted Paths. Chest. 2009;135:827-33.

3. Droogh JM, Smit M, Absalom AR, Ligtenberg JJ, Zijlstra JG. Transferring the critically ill patient: are we there yet? Crit Care. 2015;19:62.

4. Kahn JM, Linde-Zwirble WT, Wunsch H, Barnato AE, Iwashyna TJ, Roberts MS, et al. Potential Value of Regionalized Intensive Care for Mechanically Ventilated Medical Patients. Am J Respirand Crit Care Med.2008;177:285-91.

5. Jacob b Keeperman. Interfacility transportation. En: David C. Cone, Jane H. Brice, Theodore R. Delbridge, J. Brent Myers, editors. Emergency Medical Services: Clinical practice and systems oversight. $1^{\text {st }}$ edition. United Kigdom. John Wiley \& Sons, Ltd; 2015; vol.2: 29-35.

6. Raymond L. Fowler. Emergency care regionalization. En: David C. Cone, Jane H. Brice, Theodore R. Delbridge, J. Brent Myers. editores. Emergency Medical Services: Clinical practice and systems oversight.1. United Kigdom. John Wiley \& Sons, Ltd; 2015; vol.2: 123-133.

7. Combes A, Brodie D, Bartlett R, Brochard L, Brower R, Conrad S, et al. Position Paper for the Organization of Extracorporeal Membrane Oxygenation Programs for Acute Respiratory Failure in Adult Patients. Am J Respir Crit Care Med.2014;190:488-96.

8. Noah MA, Peek GJ, Finney SJ, Griffiths MJ, Harrison DA, Grieve R, et al. Referral to an extracorporeal membrane oxygenation center and mortality among patients with severe 2009 influenza A(H1N1). JAMA. 2011;306:1659-68.

9. Barbaro RP, Odetola FO, Kidwell KM, Paden ML, Bartlett RH, Davis MM, et al. Association of hospital-level volume of extracorporeal membrane oxygenation cases and mortality. Analysis of the extracorporeal life support organization registry. Am J Respir Crit Care Med. 2015;19:894-901. 
10. Maria Grazia Calabrò, Federico Pappalardo, Alberto Zangrillo. Structure of an ECMO Network for Respiratory Support. En: Fabio Sangalli?? Nicolò Patroniti, Antonio Pesenti? ? ?editors. ECMO-Extracorporeal Life Support in Adults.1st ed. Italia: Springer-Verlag; 20142.p.265-272.

11. Beurtheret S, Mordant P, Paoletti X, Marijon E, Celermajer DS, Léger P, et al. Emergency circulatory support in refractory cardiogenic shock patients in remote institutions: a pilot study (the cardiac-RESCUE program). Eur Heart J. 2013;34:112-20.

12. Combes A, Brodie D, Chen Y-S, Fan E, Henriques JPS, Hodgson C, et al. The ICM research agenda on extracorporeal life support. Intensive Care Med. 2017;43:1306-1318

13. Orr RA, Felmet KA, Han Y, McCloskey KA, Dragotta MA, Bills DM, et al. Pediatric Specialized Transport Teams Are Associated With Improved Outcomes. Pediatrics. 2009;124:40-8.

14. Peek GJ, Mugford M, Tiruvoipati R, Wilson A, Allen E, Thalanany MM, et al. Efficacy and economic assessment of conventional ventilatory support versus extracorporeal membrane oxygenation for severe adult respiratory failure (CESAR): a multicentre randomised controlled trial. Lancet.2009;374:1351-63.

15. Combes A, Leprince P, Luyt C-E, Bonnet N, Trouillet J-L, Léger P, et al. Outcomes and long-term quality-of-life of patients supported by extracorporeal membrane oxygenation for refractory cardiogenic shock. Crit Care Med.2008;36:1404-11.

16. Abrams D, Brodie D. Extracorporeal Membrane Oxygenation for Adult Respiratory Failure: 2017 Update. Chest. 2017; 152:639-649.

17. Ariza-Solé A, Sánchez-Salado JC, Lorente V, González-Costello J, Sbraga F, Cequier Á. Curva de aprendizaje y pronóstico en pacientes con shock cardiogénico refractario tratados con soporte ventricular tipo ECMO. Med Intensiva.2015;39:523-5.

18. Rihal CS, Naidu SS, Givertz MM, Szeto WY, Burke JA, Kapur NK, et al. 2015 SCAI/ACC/HFSA/STS Clinical Expert Consensus Statement on the Use of Percutaneous Mechanical Circulatory Support Devices in Cardiovascular Care (Endorsed by the American Heart Association, the Cardiological Society of India, and Sociedad Latino Americana de Cardiologia Intervencion; Affirmation of Value by the Canadian Association of Interventional Cardiology-Association Canadienne de Cardiologie d'intervention). J Card Fail.2015;2:499-518.

19. Levy B, Bastien O, Karim B, Benjelid K, Cariou A, Chouihed T, et al. Experts recommendations for the management of adult patients with cardiogenic shock. Ann Intensive Care.2015;5:52.

20. Ponikowski P, Voors AA, Anker SD, Bueno H, Cleland JGF, Coats AJS, et al. 2016 ESC Guidelines for the diagnosis and treatment of acute and chronic heart failure The Task Force for the diagnosis and treatment of acute and chronic heart failure of the European Society of Cardiology (ESC) Developed with the special contribution of the Heart Failure Association (HFA) of the ESC. Eur Heart J. 2016;37:2129-200.

21. Thiele H, Ohman EM, Desch S, Eitel I, de Waha S. Management of cardiogenic shock. Eur Heart J. 2015;36:1223-30.

22. Hernández-Tejedor A, Peñuelas O, Sirgo Rodríguez G, Llompart-Pou JA, Palencia Herrejón E, Estella A, et al. Recomendaciones para el tratamiento de los pacientes críticos de los Grupos de Trabajo de la Sociedad Española de 
Medicina Intensiva, Crítica y Unidades Coronarias (SEMICYUC). Med Intensiva. 2017;41:285-305.

23. Boedy RF, Howell CG, Kanto WP. Hidden mortality rate associated with extracorporeal membrane oxygenation. J Pediatr.1990;117:462-4.

24. Australia and New Zealand Extracorporeal Membrane Oxygenation (ANZ ECMO) Influenza Investigators, Davies A, Jones D, Bailey M, Beca J, Bellomo R, et al. Extracorporeal Membrane Oxygenation for 2009 Influenza A(H1N1) Acute Respiratory Distress Syndrome. JAMA.2009;302:1888-95.

25. Patroniti N, Zangrillo A, Pappalardo F, Peris A, Cianchi G, Braschi A, et al. The Italian ECMO network experience during the 2009 influenza A(H1N1) pandemic: preparation for severe respiratory emergency outbreaks. Intensive Care Med. 2011;37:1447-57.

26. Lucchini A, De Felippis C, Elli S, Gariboldi R, Vimercati S, Tundo P, et al. Mobile ECMO team for inter-hospital transportation of patients with ARDS: a retrospective case series. Heart Lung Vessel. 2014;6:262-73.

27. Extracorporeal Life Support Organization (ELSO). Guidelines of ECMO transport.May2015.pdf. https://www.elso.org/Portals/0/Files/ELSO\%20GUIDELINES\%20FOR\%20EC MO\%20TRANSPORT_May2015.pdf. Último acceso: 22/7/2017 1:11:35

28. Boletín Oficial del Estado (BOE). Orden FMO/2189/2010. Requisitos exigibles para la realización de las operaciones de transporte aéreo comercial por helicópteros civiles._https://www.boe.es/boe/dias/2010/08/11/pdfs/BOEA-2010-12970.pdf. Último acceso: 10/11/2017 11:01:00

29. Ericsson A, Frenckner B, Broman LM. Adverse Events during Inter-Hospital Transports on Extracorporeal Membrane Oxygenation. Prehosp Emerg Care.2017;21:448-55.

30. Broman LM, Frenckner B. Transportation of Critically Ill Patients on Extracorporeal Membrane Oxygenation. Front Pediatr. 2016;4:63.

31. Biscotti M, Agerstrand C, Abrams D, Ginsburg M, Sonett J, Mongero L, et al. One Hundred Transports on Extracorporeal Support to an Extracorporeal Membrane Oxygenation Center. The Annals of Thoracic Surgery. 2015;100:34-40.

32. Guillaume Lebreton, Hassan Debbagh, Mohamed-Ali Mnif, Dabor Résière, Robert Vignes, Bruno Sanchez et al. UMAC Antilles-Guyane : des îles entre ailes. Journal SFCTCV Société Française de Chirurgie Thoracique et CardioVasculaire. 2013;17:35-41

33. Mosier JM, Kelsey M, Raz Y, Gunnerson KJ, Meyer R, Hypes CD, et al. Extracorporeal membrane oxygenation (ECMO) for critically ill adults in the emergency department: history, current applications, and future directions. Crit Care.2015;19:431.

34. Extracorporeal Life Support Organization (ELSO). Guidelines of adult \begin{tabular}{lll} 
respiratory & failure. & August. \\
\hline
\end{tabular} https://www.elso.org/Portals/0/ELSO\%20Guidelines\%20For\%20Adult\%20R espiratory\%20Failure\%201 4.pdf. Último acceso 4/1/2018.

35. Mendes PV, de Albuquerque Gallo C, Besen BAMP, Hirota AS, de Oliveira Nardi $\mathrm{R}$, Dos Santos EV, et al. Transportation of patients on extracorporeal membrane oxygenation: a tertiary medical center experience and systematic review of the literature. Ann Intensive Care. 2017;7:14. 
36. Ramani GV, Mehra MR. Evaluación del riesgo en pacientes en estado crítico a la espera de trasplante: un paso adelante. Rev Esp Cardiol. 1 de marzo de 2011;64(03):175-6.

37. Lietz K, Miller LW. Patient selection for left-ventricular assist devices. Curr Opin Cardiol. 2009;24:246-51.

38. Schmidt M, Burrell A, Roberts L, Bailey M, Sheldrake J, Rycus PT, et al. Predicting survival after ECMO for refractory cardiogenic shock: the survival after veno-arterial-ECMO (SAVE)-score. Eur Heart J. 2015;36:2246-56.

39. Ziemba EA, John R. Mechanical Circulatory Support for Bridge to Decision: Which Device and when to Decide. J Card Surg. 2010;25:425-33.

40. Nuding S, Werdan K. IABP plus ECMO-Is one and one more than two? J Thorac Dis. 2017;9:961-4.

41. Bréchot N, Demondion P, Santi F, Lebreton G, Pham T, Dalakidis A, et al. Intraaortic balloon pump protects against hydrostatic pulmonary oedema during peripheral venoarterial-extracorporeal membrane oxygenation. European Heart Journal: Acute Cardiovascular Care. 2017;2048872617711169.

42. Guillaume Lebreton, Bruno Sanchez, Jean-Luc Hennequin, Dabor Resière, Didier Hommel, Christian Léonard, Hossein Mehdaoui and François Roques. The French airbridge for circulatory support in the Carribean. Interactive CardioVascular and Thoracic Surgery. 2012; 15:420-5

43. Charon C, Allyn J, Bouchet B, Nativel F, Braunberger E, Brulliard C, et al. Ten thousand kilometre transfer of cardiogenic shock patients on venoarterial extracorporeal membrane oxygenation for emergency heart transplantation: Cooperation between Reunion Island and Metropolitan France. Eur Heart J Acute Cardiovasc Care. 2017;2048872617719652. doi $10.1177 / 2048872617719652$

44. Walters WA, Wydro GC, Hollander T, Brister N. TRANSPORT OF THE VENTRICULAR ASSIST DEVICE-SUPPORTED PATIENT. Case series. Prehosp Emerg Care. 2005;9:90-7.

45. Matsuwaka R, Matsuda H, Kaneko M, Miyamoto Y, Masai T, Nakatani T, et al. Overseas transport of a patient with an extracorporeal left ventricular assist device. Ann Thorac Surg. 1995;59:522-3.

46. Kim SJ, Kim HJ, Lee HY, Ahn HS, Lee SW. Comparing extracorporeal cardiopulmonary resuscitation with conventional cardiopulmonary resuscitation: A meta-analysis. Resuscitation. junio de 2016;103:106-16.

47. Ortega-Deballon I, Hornby L, Shemie SD, Bhanji F, Guadagno E. Extracorporeal resuscitation for refractory out-of-hospital cardiac arrest in adults: A systematic review of international practices and outcomes. Resuscitation. abril de 2016;101:12-20.

48. Conrad SA, Rycus PT. Extracorporeal membrane oxygenation for refractory cardiac arrest. Ann Card Anaesth.2017;20:S4-10.

49. Le Guen M, Nicolas-Robin A, Carreira S, Raux M, Leprince P, Riou B, et al. Extracorporeal life support following out-of-hospital refractory cardiac arrest. Crit Care. 2011;15:R29.

50. Ha TS, Yang JH, Cho YH, Chung CR, Park C-M, Jeon K, et al. Clinical outcomes after rescue extracorporeal cardiopulmonary resuscitation for out-of-hospital cardiac arrest. Emerg Med J. 2017;34:107-11. 
51. Rojas-Peña A, Sall LE, Gravel MT, Cooley EG, Pelletier SJ, Bartlett RH, et al. Donation after circulatory determination of death: the university of michigan experience with extracorporeal support. Transplantation.2014;98:328-34.

52. Miñambres E, Suberviola B, Guerra C, Lavid N, Lassalle M, González-Castro A, et al. Experience of a Maastrich type II non heart beating donor program in a small city: preliminary results. Med Intensiva. octubre de 2015;39:433-41.

53. Pérez-Villares J.M, Lara-Rosales R.. Desplazamientos interhospitalarios de un equipo móvil para preservación de órganos con oxigenación por membrana extracorpórea en donantes en asistolia controlada. Carta Científica. Med Intensiva. 2017.doi: 10.1016/j.medin.2017.02.010

54. Biscotti M, Gannon WD, Agerstrand C, Abrams D, Sonett J, Brodie D, et al. Awake Extracorporeal Membrane Oxygenation as Bridge to Lung Transplantation: A 9-Year Experience. Ann Thorac Surg. 2017;104: 412-9.

55. Keller D, Lotz C, Kippnich M, Adami P, Kranke P, Roewer N, et al. [Venoarterial extracorporeal membrane oxygenation in an awake patient : Use of the mobile ECMO team for fulminant pulmonary embolism]. Anaesthesist. 2015;64:385-9.

56. Arcadipane A, Martucci G. Air Transport: FIxed-Wing and Helicopter. En: Fabio Sangalli, Nicolò Patronitti, Antonio Pesenti, Editors. ECMO- Extracorporeal Life Support in Adults. 1st ed. Italia: Springer-Verlag;2014.p.445-453.

57. Whiteley S, Macartney I, Mark J, Barrat HS, Binks R. Guidelines for transport of the critically ill adult. Intensive Care Society. 2011 https://www.ics.ac.uk/AsiCommon/Controls/BSA/Downloader.aspx?iDocum entStorageKey=79bdff30-7676-46dd-856db47e4d667568\&iFileTypeCode=PDF\&iFileName=Transport $\% 20$ of $\% 20$ the $\% 2$ 0Critically\%20Ill\%20Adult. Último acceso 4/1/2018.

58. Brändström H, Winsö 0 , Lindholm L, Haney M. Regional intensive care transports: a prospective analysis of distance, time and cost for road, helicopter and fixed-wing ambulances. Scandinavian Journal of Trauma, Resuscitation and Emergency Medicine. 2014;22:36.

59. Lunz D, Philipp A, Judemann K, Amann M, Foltan M, Schmid C, et al. First experience with the deltastream(R) DP3 in venovenous extracorporeal membrane oxygenation and air-supported inter-hospital transport. Interact Cardiovasc Thorac Surg.2013;17:773-7.

60. ecmo-preparation-checklist.pdf http://www.karolinska.se/globalassets/global/alb/ecmo/ecmo-preparationchecklist.pdf. Último acceso: 5/8/2017.

61. ecmo-news-and-research-ecmo_transport_system_manual.pdf https://www.hawaiipacifichealth.org/media/1482/ecmo-news-and-researchecmo transport system manual.pdf. Último acceso: 5/8/2017

62. Ventiladores Portátiles. En: Ministerio de Defensa, editor. Manual de soporte vital avanzado en combate. España: Ministerio de Defensa; 2014; p.256-269

63. Oxylog® $\quad 3000$ plus: https://www.draeger.com/es es/FireServices/Products/Ventilation-and-Respiratory-Monitoring/Emergency-andTransport-Ventilation/Oxylog-3000-plus. Último acceso 5/8/2017.

64. López M. Ventilación mecánica en pacientes tratados con membrana de oxigenación extracorpórea (ECMO). Med Intensiva 2017;41:491-6

65. Oxigenoterapia. Cálculo de consumo de oxígeno. En: Ministerio de Defensa, editor. Manual de soporte vital avanzado en combate. España: Ministerio de Defensa; 2014; p240-255 
66. García Río F, Borderías Clau L, Casanova Macario C, Celli BR, Escarrabill Sanglás J, González Mangado N, et al. Patología respiratoria y vuelos en avión. Arch Bronconeumol. 1 de febrero de 2007;43(2):101-25

67. Vicent N.Mosesso. Angus M. Jameson. Oxygenation and ventilation. En: David C. Cone, Jane H. Brice, Theodore R. Delbridge, J. Brent Myers, editors. Emergency Medical Services: Clinical practice and systems oversight. 1st ed. United Kigdom. John Wiley \& Sons, Ltd; 2015; vol.1; p. 60-66.

68. David P.Thomson. Effect of flight. En: David C. Cone, Jane H. Brice, Theodore R. Delbridge, J. Brent Myers, editors. Emergency Medical Services: Clinical practice and systems oversight. 1st ed. United Kigdom. John Wiley \& Sons, Ltd; 2015; vol.1; p. 368-371.

69. Anthony J. Franck Jr. Diving injury. En: David C. Cone, Jane H. Brice, Theodore R. Delbridge, J. Brent Myers, editors. Emergency Medical Services: Clinical practice and systems oversight. 1st ed. United Kigdom: John Wiley \& Sons, Ltd; 2015; vol.21; p. 372-378.

70. Blair L.Bigham, Daniel Patterson. Culture of patient safety. En: David C. Cone, Jane H. Brice, Theodore R. Delbridge, J. Brent Myers, editors. Emergency Medical Services: Clinical practice and systems oversight. 1st ed. United Kigdom: John Wiley \& Sons, Ltd; 2015; vol.1; p. 491-499. 
Tabla 1: Escala INTERMACS (Interagency Registry for Mechanically Assited Circulatory Support). ECMO: extracorporeal membrane oxygenation. VAD: Dispositivo asistencia ventricular.

NYHA: New York Heart Association

\begin{tabular}{|c|c|c|c|}
\hline INTERMACS & Descripción & $\begin{array}{l}\text { Tiempo para soporte } \\
\text { circulatorio }\end{array}$ & $\begin{array}{l}\text { Supervivencia } \\
\text { con soporte }\end{array}$ \\
\hline $\mathbf{1}$ & $\begin{array}{l}\text { Inestabilidad hemodinámica pese a dosis } \\
\text { crecientes de catecolaminas y/o soporte } \\
\text { circulatorio mecánico con hipoperfusión } \\
\text { crítica de órganos diana (shock cardiogénico } \\
\text { crítico) }\end{array}$ & $\begin{array}{l}\text { Soporte circulatorio a } \\
\text { corto plazo. Soporte } \\
\text { Percutáneo/ ECMO }\end{array}$ & $52 \%$ \\
\hline 2 & $\begin{array}{l}\text { Soporte inotrópico intravenoso con cifras } \\
\text { aceptables de presión arterial y deterioro } \\
\text { rápido de la función renal, el estado } \\
\text { nutricional o los signos de congestión. }\end{array}$ & $\begin{array}{l}\text { Soporte circulatorio a } \\
\text { corto plazo ECMO/VAD. } \\
\text { En días. }\end{array}$ & $63 \%$ \\
\hline 3 & $\begin{array}{l}\text { Estabilidad hemodinámica con dosis bajas o } \\
\text { intermedias de inotrópicos e imposibilidad } \\
\text { para su retirada por hipotensión, } \\
\text { empeoramiento sintomático o insuficiencia } \\
\text { renal progresiva. }\end{array}$ & VAD. Electivo en semanas. & $78 \%$ \\
\hline 4 & $\begin{array}{l}\text { Es posible retirar transitoriamente el } \\
\text { tratamiento inotrópico, pero el paciente } \\
\text { presenta recaídas sintomáticas frecuentes, } \\
\text { habitualmente con sobrecarga hídrica. }\end{array}$ & $\begin{array}{l}\text { VAD. Electivo en semanas } \\
\text { o meses. }\end{array}$ & $78 \%$ \\
\hline 5 & $\begin{array}{l}\text { Limitación absoluta de la actividad física, con } \\
\text { estabilidad en reposo, aunque habitualmente } \\
\text { con retención hídrica moderada y un cierto } \\
\text { grado de disfunción renal. }\end{array}$ & VAD, semanas o meses. & $93 \%$ \\
\hline 6 & $\begin{array}{l}\text { Menor limitación de la actividad física y } \\
\text { ausencia de congestión en reposo. Fatiga con } \\
\text { actividad ligera. }\end{array}$ & VAD a valorar & - \\
\hline 7 & $\begin{array}{l}\text { Paciente en clase funcional NYHA II-III sin } \\
\text { balance hídrico inestable. }\end{array}$ & No indicado & - \\
\hline
\end{tabular}


Tabla 2: Eventos adversos registrados durante el transporte en ECMO clasificados por causalidad y frecuencias absolutas por grupos. (29)

\begin{tabular}{|c|c|c|}
\hline $\begin{array}{c}\text { CAUSAS DE LOS EFECTOS } \\
\text { ADVERSOS }\end{array}$ & $\begin{array}{l}\text { FRECUENCIA } \\
\text { OBTENIDA }\end{array}$ & EVENTOS ADVERSOS \\
\hline Relativas al paciente & $65 \%$ & $\begin{array}{ll}\text { - } & \text { Extubación accidental } \\
\text { - } & \text { Bajo nivel de sedación } \\
\text { - Sangrados } \\
\text { - } \\
\text { - } \text { Disfunción cardiaca } \\
\text { - Recirculación } \\
\text { - Isquemia arterial } \\
\text { - Otros }\end{array}$ \\
\hline $\begin{array}{l}\text { Relativas al equipamiento } \\
\text { médico }\end{array}$ & $14,6 \%$ & $\begin{array}{ll}\text { - } & \text { Rotura de materiales } \\
\text { - } & \text { Gasto del oxígeno } \\
\text { - } & \text { Trombosis del circuito } \\
\text { - } & \text { Fallo eléctrico } \\
\text { - } & \text { Gasto de baterías } \\
\text { - } & \text { Otros }\end{array}$ \\
\hline $\begin{array}{l}\text { Relativas al personal } \\
\text { médico }\end{array}$ & $5,8 \%$ & $\begin{array}{ll}\text { - } & \text { Fallos de comunicación } \\
\text { - Olvidos de material } \\
\text { - } \\
\text { - } \text { Falta de personal } \\
\end{array}$ \\
\hline $\begin{array}{l}\text { Relativas al medio de } \\
\text { transporte }\end{array}$ & $12,6 \%$ & $\begin{array}{l}\text { - Fallos de logística } \\
\text { - Ambulancia errónea } \\
\text { - Tráfico } \\
\text { - Fallos del suministro eléctrico } \\
\text { - Otros }\end{array}$ \\
\hline Causas ambientales & $1,9 \%$ & $\begin{array}{l}\text { - Congelación de las vías venosas. } \\
\text { - Otros }\end{array}$ \\
\hline
\end{tabular}


Tabla 3: Eventos adversos registrados durante el transporte en ECMO clasificados por niveles de gravedad, frecuencias absolutas y análisis por subgrupos según la causalidad. (29)

\begin{tabular}{|c|c|c|c|}
\hline $\begin{array}{l}\text { GRADUACIÓN } \\
\text { DEL RIESGO }\end{array}$ & DEFINICIÓN & $\%$ & $\begin{array}{c}\text { ANÁLISIS POR SUBGRUPOS } \\
\text { SEGÚN CAUSAS }\end{array}$ \\
\hline Categoría 1 & $\begin{array}{l}\text { Repercusión en la } \\
\text { mortalidad o alta } \\
\text { morbilidad si no es } \\
\text { resuelto en cuestión de } \\
\text { segundos. }\end{array}$ & $7,8 \%$ & $\begin{array}{l}\text { 19\% Paciente } \\
50 \% \text { Equipamiento médico } \\
\text { 19\% Personal médico } \\
12 \% \text { Causa ambiental }\end{array}$ \\
\hline Categoría 2 & $\begin{array}{l}\text { Repercusión en la } \\
\text { mortalidad o alta } \\
\text { morbilidad si no es } \\
\text { resuelto en minutos. }\end{array}$ & $58,7 \%$ & $\begin{array}{l}\text { 93\% Paciente } \\
\text { 2,5\% Equipamiento médico } \\
\text { 2,5\% Personal médico } \\
\text { 1,7\% Causa ambiental }\end{array}$ \\
\hline Categoría 3 & \begin{tabular}{l}
\multicolumn{3}{l}{ Riesgo intermedio que no } \\
produce un riesgo \\
inminente \\
morbimortalidad, pero \\
precisa de una solución.
\end{tabular} & $22,8 \%$ & $\begin{array}{l}\text { 34\% Paciente } \\
\text { 19\% Equipamiento médico } \\
\text { 13\% Personal médico } \\
\text { 34\% Medio de transporte }\end{array}$ \\
\hline Categoría 4 & $\begin{array}{l}\text { Bajo riesgo, se trata de un } \\
\text { evento a lidiar durante el } \\
\text { traslado }\end{array}$ & $10,7 \%$ & $\begin{array}{l}\text { 9\% Paciente } \\
\text { 45\% Equipamiento médico } \\
\text { 45\% Medio de Transporte }\end{array}$ \\
\hline
\end{tabular}


MOTIVOS DE ACTIVACIÓN AL EQUIPO ECMO:

$\square I N S U F I C I E N C I A$ RESPIRATORIA HIPOXEMICA O GLOBAL REFRACTARIA.

$\square$ Establecer criterios de activación del equipo ECMO.

$\square$ SHOCK CARDIOGÉNICO REFRACTARIO

$\square$ Establecer criterios de activación del equipo ECMO

REAGUDIZACIÓN DE PACIENTE EN LISTA DE TRASPLANTE

$\square$ CARDIACO (valorar criterios y tipo de soporte)

$\square$ PULMONAR (valorar criterios y tipo de soporte)

CONTRAINDICACIONES DE ECMO: Ventilación mecánica > 7días, Edad> 75 años, Peso > 150 kg,

\section{CONDICIONES NECESARIAS EN EL HOSPITAL DE ORIGEN:}

- Habitación privada si es posible.

- Información de la familia y disponibilidad para recibir información del Equipo ECMO.

- Espacio para colocar el material y sus conexiones de gases y eléctricas.

\section{INFORMACIÓN CLÍNICA IMPORTANTE:}

- ESCALA COMA DE GLASGOW (ECG) ÚLTIMA RECOGIDA.

- PARÁMETROS DE MONITORIZACIÓN: TAS, TAD, TAM, Temperatura, Balance hídrico durante el ingreso.

- PARÁMETROS RESPIRADOR: FiO2, Vtidal, FR, PEEP, Pplat

- OTRAS MEDIDAS RESPIRATORIAS: INO, Prono...

- INOTROPOS, VASOPRESORES Y DOSIS

- Presencia o no de otros soportes: TCRR, BIAC..

\section{PRUEBAS DIAGNÓSTICAS NECESARIAS:}

- EXTRACCIÓN DE ANALÍTICA 60-30 MINUTOS ANTES DE LA LLEGADA DEL EQUIPO:

- HEMOGRAMA.

- COAGULACION COMPLETA

- BIOQUÍMICA COMPLETA

- GASOMETRÍA ARTERIAL Y VENOSA

- PRUEBAS DE IMAGEN A VALORAR PREVIAS A LA IMPLANTACIÓN:

- TC TORAX

- TC CRANEAL

- ECOCARDIOGRAMA TRANSESOFÁGICO

- SOLICITAR PRUEBAS CRUZADAS Y RESERVAR:

○ SANGRE

- PLAQUETAS (a valorar)

- PLASMA (a valorar)

- SEROLOGÍAS VÍRICAS

\section{EN CASO DE IMPLANTAR LA ECMO, MATERIAL NECESARIO A PREPARAR POR PARTE} DEL HOSPITAL DE ORIGEN:

- Material quirúrgico, mesas quirúrgicas, aspiración, iluminación.

- Ecógrafo transesofágico.

\section{EN CASO DE TRANSPORTE AEREO CON NECESIDAD DE TRANSPORTE TERRESTRE:}

- Es necesario un transporte terrestre con capacidad para 4 personas, camilla, paciente y material, desde el helipuerto o aeropuerto hasta el hospital.

Figura 1: Propuesta de Checklist a realizar por el Hospital de Origen: criterios de activación del equipo ECMO, datos clínicos, analíticos y pruebas de imagen relevantes, necesidades instrumentales y de espacio para colocación de soporte extracorpóreo (53). TAS: tensión arterial sistólica; TAD: tensión arterial diastólica, TAM: tensión arterial media; FR: frecuencia respiratoria, iNO: óxido nítrico, TCRR: terapias continuas de remplazo renal; BIAC: balón de contrapulsación. 


\section{TRANSPORTE EN ECMO \\ CHECK LIST DEL EQUIPO ECMO}

MATERIAL DE TRANSPORTE Y ECMO: Comprobadas baterías cargadas y cableado presente

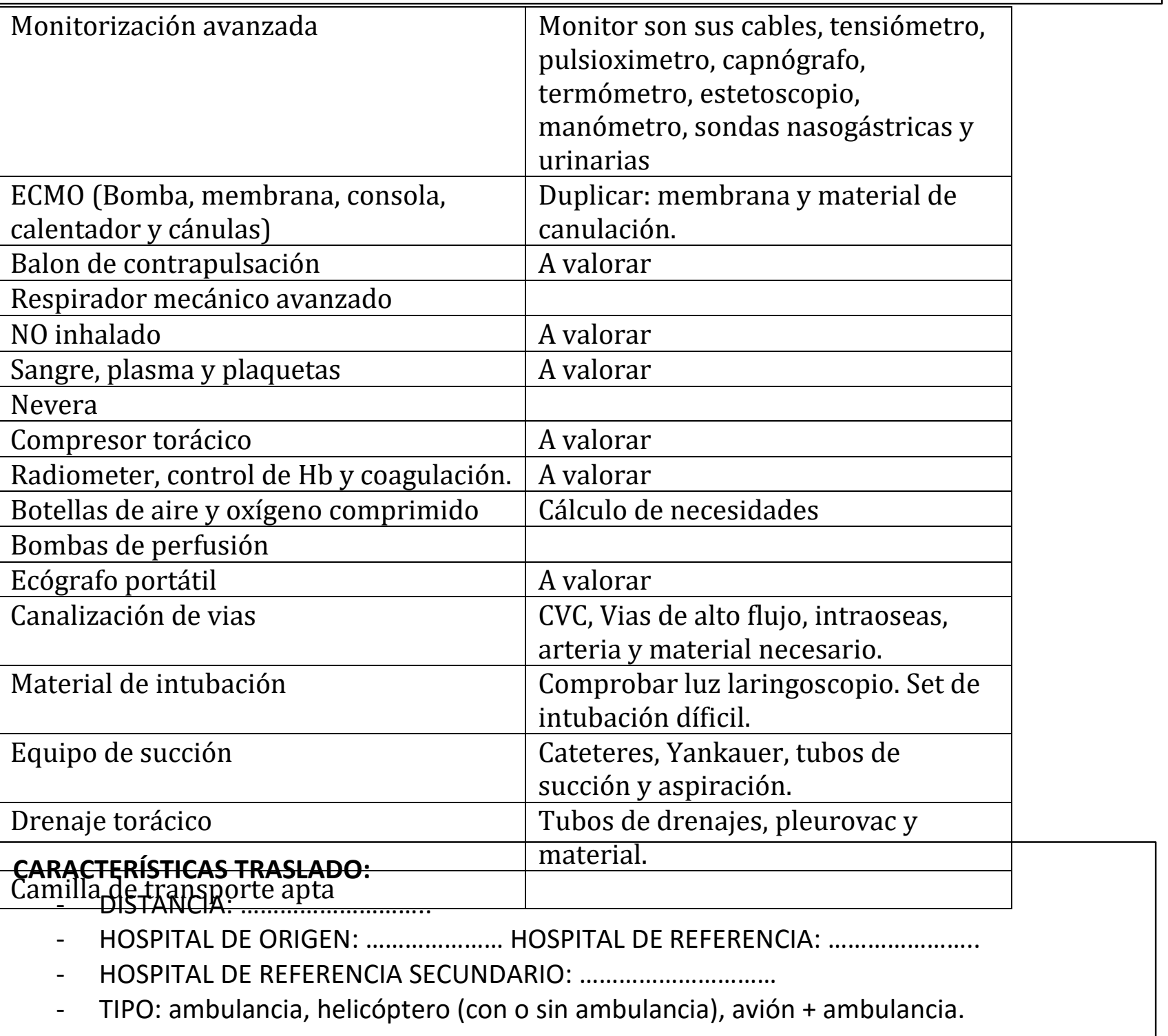

TELÉFONOS DE INTERÉS: hospital de origen, hospital secundario, ambulancia receptora, helipuerto...

VALORACIÓN CLÍNICA: CRITERIOS DE SOPORTE EXTRACORPÓREO (A Establecer por el Equipo)

- CRITERIOS ECCO 2 R, ECMO-VV

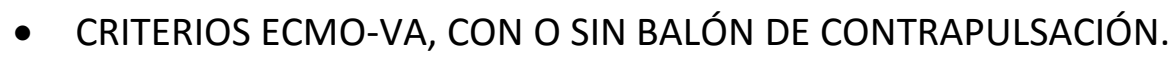

INFORMACIÓN A LA FAMILIA Y CONSENTIMIENTO INFORMADO.

CRITERIOS DE ESTABILIDAD PARA TRASLADO: A establecer.

TRANSPORTE AÉREO:

- $\mathrm{FIO}_{2}$ RESPIRADOR POST-IMPLANTACIÓN ECMO VV:

- ALTURA DE VUELO MÁXIMA:

MEDIA:

- TIEMPO DE AUTONOMÍA:

- MATERIAL FIJADO CORRECTAMENTE, TEMPERATURA DE CABINA

- AÉREO: TAPONES EN OIDOS, POSICION CORRECTA, CABIDADES AÉREAS DRENADAS.

Figura 2: Propuesta de Checklist por parte del equipo ECMO en el hospital de origen. CVC: catéter de via central. ECMO: extracorporeal membrane oxygenation. VV: veno-venosa. VA: veno-arterial ECCO2R: Extracorporeal $\mathrm{CO}_{2} \mathrm{Removal}$ 